ANNALES

POLONICI MATHEMATICI

85.3 (2005)

\title{
Coproducts and the additivity of the Szymczak index
}

\author{
by Kinga Stolot (Kraków)
}

\begin{abstract}
We prove that the index defined by Szymczak in [9] has an additivity property. Moreover we give an abstract theorem for extending coproducts from an initial category to the Szymczak category, which provides a setting for the proof of additivity.
\end{abstract}

1. Introduction. The Conley index is a topological invariant suitable to detect and investigate the properties of isolated invariant sets. Initially defined by Conley for continuous dynamical systems, it was developed by numerous authors and extended to the discrete, and recently also to the multi-valued discrete setting.

The discrete extension defined by Szymczak [9] is considered to be the most general among single-valued ones. One would expect it to have the same major properties as the classical Conley index for flows. Although Conley writes in [1] that it is obvious that the index of the disjoint union of two isolated invariant sets is the sum of their indices, so far this property has not been proved for the Szymczak index.

The additivity property appears to be more complicated in the discrete than in the continuous case. The first and obvious question is how to define a sum of indices which are objects of an abstract Szymczak category.

The main results of this paper are Theorems 8 and 12. The first answers the above question, the second is actually a statement of the additivity property of the Szymczak index.

In the abstract and elegant language of category theory a "sum of objects" is called a coproduct. Theorem 8 provides a procedure of transferring coproducts from an initial category to the category of endomorphisms and then to the Szymczak category, bearing the actual index. Roughly speaking, Theorem 12 is an application of Theorem 8 to the concrete category which is used in the definition of the Szymczak index.

2000 Mathematics Subject Classification: Primary 37B30.

Key words and phrases: discrete dynamical system, invariant set, index pair, Szymczak index, category with coproducts.

Research supported by KBN Grant No. 2 P03A 04124. 
As the index itself has a more complex structure in the discrete than in the continuous case, one would expect that the proof of additivity is more complicated. For simplicity, part of the proof devoted to carrying over the coproducts is done in a more abstract setting.

Theorem 8 also provides some information on the possibility of proving the additivity property of other indices, defined with the use of the Szymczak functor (for example those defined in [6] and [7]). Because of Theorem 8 we know that to solve this problem one needs to check if the initial category in these constructions has finite coproducts.

Finally, one should mention that in [8] Szymczak considers a special kind of index for decompositions of isolated invariant sets, mainly for the purpose of detecting periodic orbits and chaos, but does not actually deal with the additivity property.

Acknowledgements. The author would like to express her gratitude to her PhD supervisor Prof. Marian Mrozek for his suggestion to undertake this problem and assistance.

2. Preliminaries. By $\mathbb{Z}$ and $\mathbb{N}$ we denote respectively the set of all integers and the natural numbers with zero.

Let $X$ be a topological space. For a set $A \subset X$ we denote by $\operatorname{int} A$, bd $A$, $\operatorname{cl} A$ respectively the interior, boundary and closure of the set $A$. Throughout the paper by a pair of subsets of a topological space $X$ we understand a pair $(A, B)$ such that $B \subset A \subset X$.

2.1. Categories and coproducts. Let us recall after [2], [5] the notion of coproduct in a category $\mathcal{E}$.

By $\mathcal{E}(A, B)$ we denote the set of all morphisms from the object $A$ to the object $B$ in the category $\mathcal{E}$. Once the category is clear from the context, instead of $a \in \mathcal{E}(A, B)$ we will write

$$
a: A \rightarrow B .
$$

Let us stress that this notation does not imply that the morphism is necessarily a function.

For the purpose of Conley index theory it is sufficient to consider finite coproducts.

Definition 1. Let $\left\{A_{i}\right\}_{i \in I}$ be a finite family of objects of the category $\mathcal{E}$. The coproduct of $\left\{A_{i}\right\}_{i \in I}$ is a pair $\left(\bigvee_{i \in I} A_{i},\left\{\kappa_{i}: A_{i} \rightarrow \bigvee_{i \in I} A_{i}\right\}_{i \in I}\right)$ such that $\bigvee_{i \in I} A_{i} \in \mathcal{E}, \kappa_{i} \in \mathcal{E}\left(A_{i}, \bigvee_{i \in I} A_{i}\right)$ for $i \in I$ and the following condition is satisfied: for all $B \in \mathcal{E}$ and all $\phi_{i} \in \mathcal{E}\left(A_{i}, B\right)$ for $i \in I$ there is a unique $\xi \in \mathcal{E}\left(\bigvee_{i \in I} A_{i}, B\right)$ such that the following diagrams commute for all $i \in I$ : 


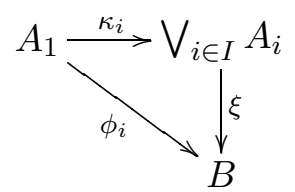

We call the morphisms $\kappa_{i}$ appearing in this definition the inclusions associated with the coproduct of the objects $\left\{A_{i}\right\}_{i \in I}$ in the category $\mathcal{E}$.

If for any finite family of objects of the category $\mathcal{E}$ there exists a coproduct we say that the category $\mathcal{E}$ has finite coproducts. The following remark (see [5]) justifies the notation $\bigvee_{i \in I} A_{i}$.

REMARK 2. If the category has finite coproducts then they are uniquely determined up to an isomorphism.

The object $\bigvee_{i \in I} A_{i}$ given by Definition 1 is called briefly a coproduct. Moreover the morphism $\xi$ uniquely determined by the family $\left\{\phi_{i}\right\}_{i \in I}$ is denoted by

$$
\vee_{i \in I} \phi_{i}:=\xi
$$

We denote by top • the category of pointed topological spaces with base point preserving maps. Objects of top $\bullet$ are $\left(X, x_{0}\right)$, where $X$ is a topological space and $x_{0} \in X$ is a base point. By $[f]_{h}$ we denote the homotopy

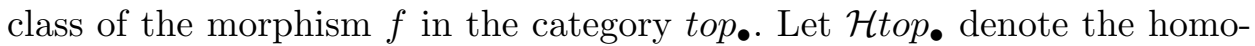

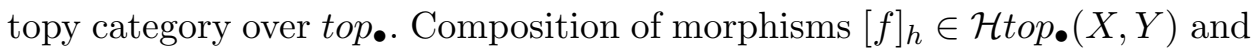
$[g]_{h} \in \mathcal{H}$ top $_{\bullet}(Y, Z)$ is denoted by

$$
[g]_{h} \bullet[f]_{h}=[g \circ f]_{h} .
$$

2.2. Endomorphism and Szymczak categories. Recall after [3] and [9] the notions of the category of endomorphisms and Szymczak category.

Let $\mathcal{E}$ be any category. Objects of the category of endomorphisms over $\mathcal{E}$ (denoted by $\operatorname{Endo}(\mathcal{E})$ ) are pairs $(A, a)$, where $A \in \mathcal{E}$ and $a \in \mathcal{E}(A, A)$. A morphism in $\operatorname{Endo}(\mathcal{E})$ from $(A, a)$ to $(B, b)$ is a morphism $\phi \in \mathcal{E}(A, B)$ for which the following diagram commutes:

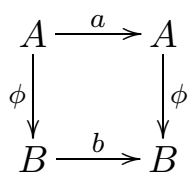

The $S z y m c z a k$ category $S z(\mathcal{E})$ has the same objects as $E n d o(\mathcal{E})$. To define the morphisms of $S z(\mathcal{E})$ let us first introduce a relation in $\operatorname{Endo}(\mathcal{E}) \times \mathbb{N}$. Let $\phi, \phi^{\prime} \in \operatorname{Endo}(\mathcal{E})((A, a),(B, b))$ and $m, m^{\prime} \in \mathbb{N}$. Then

$$
(\phi, m) \equiv\left(\phi^{\prime}, m^{\prime}\right) \Leftrightarrow \exists k \in \mathbb{N}: \phi \circ a^{m^{\prime}+k}=\phi^{\prime} \circ a^{m+k} .
$$

Morphisms in $S z(\mathcal{E})$ are, by definition,

$$
S z(\mathcal{E})((A, a),(B, b)):=\operatorname{Endo}(\mathcal{E}) \times \mathbb{N} / \equiv .
$$


The composition of the morphisms $[\phi, m]_{\equiv} \in S z(\mathcal{E})((A, a),(B, b))$ and $[\psi, n]_{\equiv} \in S z(\mathcal{E})((B, b),(C, c))$ is defined by

$$
[\psi, n]_{\equiv} \star[\phi, m]_{\equiv}=[\psi \circ \phi, n+m]_{\equiv} .
$$

2.3. Definition of the Szymczak index. In this section we recall after [9] the definition of the Szymczak index.

Assume that $X$ is a locally compact metric space, and $f: X \rightarrow X$ a continuous map. For any $A \subset X$ and $n \in \mathbb{N}$ define the invariant part of $A$ under $f$ as

$\operatorname{Inv}(A, f):=\left\{x \in A\right.$ : there exists a sequence $\left\{x_{n}\right\}_{n \in \mathbb{Z}}, x_{n} \in A$ for all $n \in \mathbb{Z}$, such that $x_{0}=x$ and $x_{n+1}=f\left(x_{n}\right)$ for all $\left.n \in \mathbb{Z}\right\}$.

A compact set $N \subset X$ satisfying

$$
\operatorname{Inv}(N, f) \subset \operatorname{int} N
$$

is called an isolating neighborhood and $S=\operatorname{Inv}(N, f)$ is called an isolated invariant set with respect to $f$.

Throughout this paper we will be using index pairs and index maps in the sense of Szymczak (the definitions below come from [9]).

Definition 3. A pair $P=\left(P_{1}, P_{2}\right)$ of compact sets such that $P_{2} \subset P_{1} \subset$ $X$ is called an index pair for an isolated invariant set $S$ with respect to $f$ if the following conditions are satisfied:

(a) $S=\operatorname{Inv}\left(\operatorname{cl}\left(P_{1} \backslash P_{2}\right), f\right) \subset \operatorname{int} P_{1} \backslash P_{2}$,

(b) if $x \in P_{2}$ then $f(x) \notin P_{1} \backslash P_{2}$,

(c) if $x \in P_{1}$ and $f(x) \notin P_{1}$ then $x \in P_{2}$.

Fundamental for the definition of the Szymczak index is the following proposition (see e.g. [4]).

TheOREM 4. For any isolated invariant set there exists an index pair.

Given any two closed subsets $P_{2} \subset P_{1} \subset X$ we denote by $P_{1} / P_{2}$ the pointed topological space $\left(P_{1} \backslash P_{2} \cup\left\{p_{2}\right\}, p_{2}\right)$, where $p_{2}$ is either any point of $P_{2}$ if $P_{2} \neq \emptyset$, or an added distinguished point $p_{2} \notin P_{1}$ otherwise. The elements of $P_{1} / P_{2}$ are denoted by $[x]$ for $x \in P_{1} \backslash P_{2} \cup\left\{p_{2}\right\}$.

If $P_{2} \neq \emptyset$ then the topology of $P_{1} / P_{2}$ is the quotient topology given by the projection $p: P_{1} \rightarrow P_{1} / P_{2}$ where

$$
p(x):= \begin{cases}{[x]} & \text { if } x \in P_{1} \backslash P_{2}, \\ {\left[p_{2}\right]} & \text { if } x \in P_{2} .\end{cases}
$$

In case $P_{2}=\emptyset$ the topology of $P_{1} \cup\left\{p_{2}\right\}$ is that of $P_{1}$ with added isolated point $p_{2}$. Note that this definition covers the case of both $P_{1}=P_{2}=\emptyset$. 
For an isolated invariant set $S$ with respect to $f$ and an associated index pair $P$ let us define the index map

$$
f_{P}: P_{1} / P_{2} \rightarrow P_{1} / P_{2}
$$

as follows:

$$
f_{P}([x]):= \begin{cases}{[f(x)]} & \text { if } x, f(x) \in P_{1} \backslash P_{2}, \\ {\left[p_{2}\right]} & \text { otherwise. }\end{cases}
$$

Due to [9, Lemma 4.3] the index map is continuous.

Definition 5. The Szymczak index of an isolated invariant set $S$ for a discrete dynamical system $f$ is the class of objects isomorphic in the category $S z\left(\mathcal{H} t_{o p} \bullet\right)$ to $\left(P_{1} / P_{2},\left[f_{P}\right]_{h}\right)$, where $P=\left(P_{1}, P_{2}\right)$ is any index pair for $S$.

The class of such isomorphic objects is denoted by $C(S, f)$. By [9, Proposition 2.2], it does not depend on the choice of the specific index pair.

3. Carrying over the coproducts. We now state the crucial result of this paper, which provides an abstract setting for proving the additivity property of indices of Conley type. Namely assuming that $\mathcal{E}$ is a category with finite coproducts we prove that both

(i) $\operatorname{Endo}(\mathcal{E})$ has finite coproducts,

(ii) $S z(\mathcal{E})$ has finite coproducts.

For later purposes we state this theorem in a more detailed form, giving the actual formulas for coproducts in the above categories. Before we state the theorem we prove two lemmas.

Throughout this section $\{A\}_{i \in I}$ is a finite family of objects of $\mathcal{E}$ and $a_{i} \in \mathcal{E}\left(A_{i}, A_{i}\right)$ for $i \in I$. The following is obvious from the definition of the Szymczak relation.

Lemma 6. If $\left[\mu_{i}, n_{i}\right]_{\equiv} \in S z(\mathcal{E})\left(\left(A_{i}, a_{i}\right),(C, c)\right)$ for $i \in I$ and $n:=$ $\max \left\{n_{i}: i \in I\right\}$, then

$$
\forall i \in I:\left[\mu_{i}, n_{i}\right]_{\equiv}=\left[\mu_{i} \circ a_{i}^{n-n_{i}}, n\right]_{\equiv} .
$$

Lemma 7. Assume $\mathcal{E}$ is a category with finite coproducts. Let

$$
\alpha:=\vee_{i \in I}\left(\kappa_{i} \circ a_{i}\right) \in \mathcal{E}\left(\bigvee_{i \in I} A_{i}, \bigvee_{i \in I} A_{i}\right),
$$

where the $\kappa_{i}$ appear in the definition of the coproduct. Then

$$
\forall i \in I \forall k \in \mathbb{N} \backslash\{0\}: \quad \kappa_{i} \circ a_{i}^{k}=\alpha^{k} \circ \kappa_{i} .
$$

Proof. Take any $i \in I$. The assertion follows easily by induction on $k \in \mathbb{N}$. If $k=1$ it is obvious from the definition of the coproduct. Assuming that $\kappa_{i} \circ a_{i}^{k}=\alpha^{k} \circ \kappa_{i}$ we obtain

$$
\kappa_{i} \circ a_{i}^{k+1}=\left(\kappa_{i} \circ a_{i}^{k}\right) \circ a_{i}=\left(\alpha^{k} \circ \kappa_{i}\right) \circ a_{i}=\alpha^{k} \circ\left(\alpha \circ \kappa_{i}\right)=\alpha^{k+1} \circ \kappa_{i} .
$$


THEOREM 8. Assume that $\mathcal{E}$ is a category with finite coproducts.

(i) If $\left\{\left(A_{i}, a_{i}\right)\right\}_{i \in I}$ is a finite family of objects of the category Endo $(\mathcal{E})$, then

$$
\left(\left(\bigvee_{i \in I} A_{i}, \alpha\right),\left\{\kappa_{i}\right\}_{i \in I}\right)
$$

is their coproduct, where the morphism $\alpha$ is defined by formula (10) and $\kappa_{i} \in \operatorname{Endo}(\mathcal{E})\left(\left(A_{i}, a_{i}\right),\left(\bigvee_{i \in I} A_{i}, \alpha\right)\right)$ for $i \in I$.

(ii) The coproduct of the family $\left\{\left(A_{i}, a_{i}\right)\right\}_{i \in I}$ in the category $S z(\mathcal{E})$ is

$$
\left(\left(\bigvee_{i \in I} A_{i}, \alpha\right),\left\{\left[\kappa_{i}, 0\right]_{\equiv}\right\}_{i \in I}\right),
$$

where $\alpha$ is as in $(\mathrm{i})$ and $\left[\kappa_{i}, 0\right]_{\equiv} \in S z(\mathcal{E})\left(\left(A_{i}, a_{i}\right),\left(\bigvee_{i \in I} A_{i}, \alpha\right)\right)$.

Proof. (i) Consider the morphisms

$$
\phi_{i}:=\kappa_{i} \circ a_{i} \in \mathcal{E}\left(A_{i}, \bigvee_{i \in I} A_{i}\right) \quad \text { for } i \in I .
$$

Because $\mathcal{E}$ by assumption has coproducts, there exists exactly one morphism, denoted by $\alpha$ and defined by formula (10), which satisfies

$$
\alpha \circ \kappa_{i}=\phi_{i} \text {. }
$$

We wish to show that $(11)$ is a coproduct of the family $\left\{\left(A_{i}, a_{i}\right)\right\}_{i \in I}$ in $\operatorname{Endo}(\mathcal{E})$. In order to prove that $\kappa_{i}:\left(A_{i}, a_{i}\right) \rightarrow\left(\bigvee_{i \in I} A_{i}, \alpha\right)$ is a morphism in $\operatorname{Endo}(\mathcal{E})$ for any $i \in I$ it is enough to note that due to (13) and (14) the following diagram commutes:

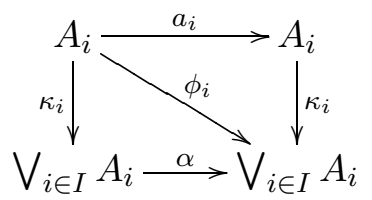

Let us check if the pair (11) satisfies condition (2) from the definition of the coproduct. Let $(C, c)$ be any object in $\operatorname{Endo}(\mathcal{E})$ and let

$$
\mu_{i} \in \operatorname{Endo}(\mathcal{E})\left(\left(A_{i}, a_{i}\right),(C, c)\right) \quad \text { for } i \in I
$$

be any family of morphisms. Then the following family of diagrams for $i \in I$ commutes:

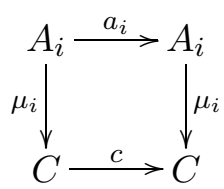

We will show that there exists exactly one morphism

$$
\xi \in \operatorname{Endo}(\mathcal{E})\left(\left(\bigvee_{i \in I} A_{i}, \alpha\right),(C, c)\right)
$$


such that the following diagrams commute for $i \in I$ :

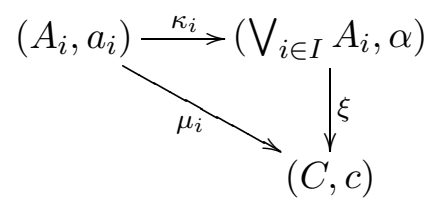

Because $C \in \mathcal{E}$ and $\mu_{i} \in \mathcal{E}\left(A_{i}, C\right)$ for $i \in I$, from the definition of the coproduct in $\mathcal{E}$ there exists exactly one morphism $\vee_{i \in I} \mu_{i} \in \mathcal{E}\left(\bigvee_{i \in I} A_{i}, C\right)$ such that the following diagrams commute for $i \in I$ :

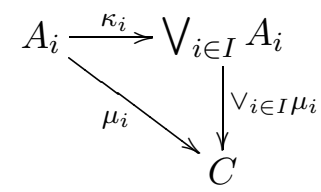

Let us first check if $\vee_{i \in I} \mu_{i}$ is also a morphism in the category $\operatorname{Endo}(\mathcal{E})$ from the object $\left(\bigvee_{i \in I} A_{i}, \alpha\right)$ to $(C, c)$, i.e. if the following diagram commutes:

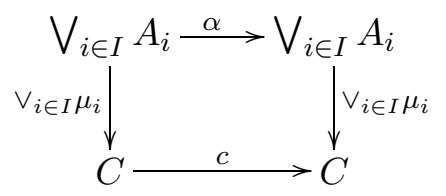

Notice that joining diagrams (19) and (16) we find that the following diagram commutes:

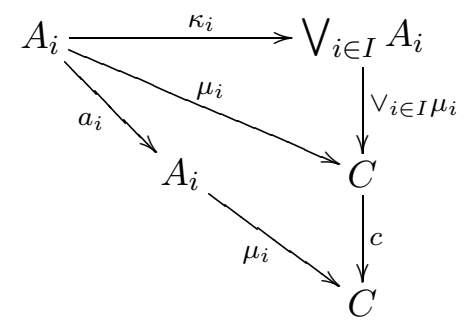

Similarly joining diagrams (15) and (19) we find the following commuting diagram:

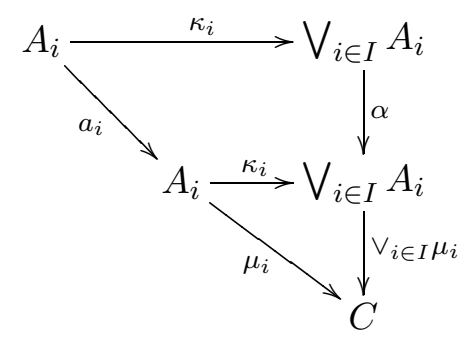

The vertical compositions in (21) and (22) are equal, from the uniqueness of the morphism in the definition of the coproduct in the category $\mathcal{E}$ for the 
family $\left\{\mu_{i} \circ a_{i}\right\}_{i \in I}$. The resulting equality

$$
c \circ \vee_{i \in I} \mu_{i}=\left(\vee_{i \in I} \mu_{i}\right) \circ \alpha
$$

gives the required commutativity of (20).

To complete the proof of (i) put

$$
\xi:=\vee_{i \in I} \mu_{i}
$$

Commutativity of (18) for $i \in I$ is a consequence of the fact that (19) commutes for $i \in I$.

It remains to show that (23) is the unique morphism that satisfies condition (18). Suppose $\widetilde{\xi} \in \operatorname{Endo}(\mathcal{E})\left(\left(\bigvee_{i \in I} A_{i}, \alpha\right),(C, c)\right)$ is another such morphism. Then $\widetilde{\xi}: \bigvee_{i \in I} A_{i} \rightarrow C$ is a morphism in $\mathcal{E}$ for which the following diagram commutes:

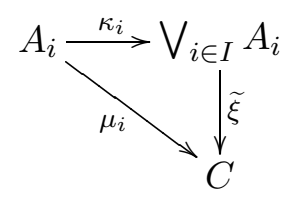

Now $\xi=\widetilde{\xi}$ from the definition of the coproducts in $\mathcal{E}$.

(ii) As the objects of $\operatorname{Endo}(\mathcal{E})$ and $S z(\mathcal{E})$ are the same, we can proceed to checking if the pair (12) satisfies condition (2) from the definition of the coproduct in $S z(\mathcal{E})$. Let $(C, c)$ be any object of $S z(\mathcal{E})$ and let

$$
\left[\mu_{i}, n_{i}\right]_{\equiv} \in S z(\mathcal{E})\left(\left(A_{i}, a_{i}\right),(C, c)\right) \quad \text { for } i \in I
$$

be any finite family of morphisms. Let $n:=\max \left\{n_{i}: i \in I\right\}$. Denote by

$$
\xi:=\vee_{i \in I}\left(\mu_{i} \circ a_{i}^{n-n_{i}}\right)
$$

the morphism which exists from the definition of the coproduct in $\operatorname{Endo}(\mathcal{E})$. We will show that

$$
[\xi, n]_{\equiv} \in S z(\mathcal{E})\left(\left(\bigvee_{i \in I} A_{i}, \alpha\right),(C, c)\right)
$$

satisfies the condition from Definition 1 . We first prove that the following diagrams for $i \in I$ commute in the Szymczak category:

$$
\left(A_{i}, a_{i}\right) \stackrel{\left[\kappa_{i}, 0\right] \equiv}{\longrightarrow}\left(\bigvee_{i \in I} A_{i}, \alpha\right)
$$

From the definition of the coproduct in $\operatorname{Endo}(\mathcal{E})$ the following diagrams commute for $i \in I$ : 


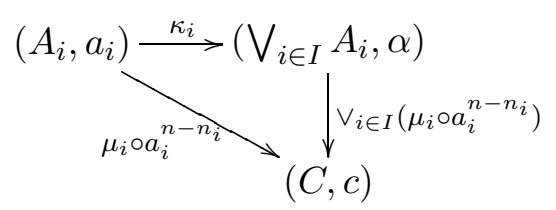

From Lemma 6 we obtain

$$
\left[\mu_{i} \circ a_{i}^{n-n_{i}}, n\right]_{\equiv}=\left[\mu_{i}, n_{i}\right]_{\equiv},
$$

and from (25) and the commutativity of (28) it follows that

$$
\left[\xi \circ \kappa_{i}, n\right]_{\equiv}=\left[\mu_{i}, n_{i}\right]_{\equiv} .
$$

This shows that diagram (27) commutes for $i \in I$.

It remains to show that (26) is the unique morphism which satisfies the requirements of Definition 1. Suppose that

$$
[\widetilde{\xi}, m]_{\equiv} \in S z(\mathcal{E})\left(\left(\bigvee_{i \in I} A_{i}, \alpha\right),(C, c)\right)
$$

is also a morphism satisfying (27). Then

$$
\left[\widetilde{\xi} \circ \kappa_{i}, m\right]_{\equiv}=\left[\mu_{i}, n_{i}\right]_{\equiv},
$$

therefore using (29) we obtain

$$
\left[\xi \circ \kappa_{i}, n\right]_{\equiv}=\left[\widetilde{\xi} \circ \kappa_{i}, m\right]_{\equiv},
$$

and from the definition of the Szymczak relation

$$
\exists s \in \mathbb{N}: \quad \xi \circ \kappa_{i} \circ a_{i}^{m+s}=\widetilde{\xi} \circ \kappa_{i} \circ a_{i}^{n+s} .
$$

Applying Lemma 7 twice we obtain, for any $i \in I$,

$$
\xi \circ \kappa_{i} \circ a_{i}^{m+s}=\xi \circ \alpha^{m+s} \circ \kappa_{i}, \quad \widetilde{\xi} \circ \kappa_{i} \circ a_{i}^{n+s}=\widetilde{\xi} \circ \alpha^{n+s} \circ \kappa_{i} .
$$

By using the above equalities formula (31) reads

$$
\exists s \in \mathbb{N}: \quad \xi \circ \alpha^{m+s} \circ \kappa_{i}=\widetilde{\xi} \circ \alpha^{n+s} \circ \kappa_{i},
$$

for any $i \in I$. From the definition of $\equiv$ this is equivalent to $[\xi, n]_{\equiv}=[\widetilde{\xi}, m]_{\equiv}$, which completes the proof.

4. Coproducts in the category $\mathcal{H} t o p$. . We show that the category $\mathcal{H} t o p \bullet$ has finite coproducts. Then we give a precise formula for the coproduct both in $\mathcal{H} t o p_{\bullet}$ and in $S z\left(\mathcal{H} t o p_{\bullet}\right)$.

To simplify the notation we only deal with coproducts of two objects. The formula obviously extends to the coproduct of any finite number of objects.

Theorem 9. Let $\left(X, x_{0}\right)$ and $\left(Y, y_{0}\right)$ be any objects of $\mathcal{H}$ top.. Then the coproduct of $\left(X, x_{0}\right)$ and $\left(Y, y_{0}\right)$ is the pair

$$
\left(\left(X, x_{0}\right) \vee\left(Y, y_{0}\right),\left\{\left[\kappa_{X}\right]_{h},\left[\kappa_{Y}\right]_{h}\right\}\right),
$$


where

$$
\left(X, x_{0}\right) \vee\left(Y, y_{0}\right):=\left(X \times\left\{y_{0}\right\} \cup\left\{x_{0}\right\} \times Y,\left(x_{0}, y_{0}\right)\right)
$$

is the object of $\mathcal{H}$ top . and the associated inclusions are defined as the pointed homotopy classes of the maps

$$
\kappa_{X} \in \operatorname{top}_{\bullet}\left(\left(X, x_{0}\right),\left(X, x_{0}\right) \vee\left(Y, y_{0}\right)\right)
$$

with $\kappa_{X}(x):=\left(x, y_{0}\right)$ for any $x \in X$ and

$$
\kappa_{Y} \in \operatorname{top}_{\bullet}\left(\left(Y, y_{0}\right),\left(X, x_{0}\right) \vee\left(Y, y_{0}\right)\right)
$$

with $\kappa_{Y}(y):=\left(x_{0}, y\right)$ for any $y \in Y$.

Proof. Consider any object $\left(Z, z_{0}\right) \in \mathcal{H}$ top • and any morphisms

$$
[f]_{h} \in \mathcal{H} \text { top }_{\bullet}\left(\left(X, x_{0}\right),\left(Z, z_{0}\right)\right), \quad[g]_{h} \in \mathcal{H} t o p_{\bullet}\left(\left(Y, y_{0}\right),\left(Z, z_{0}\right)\right) .
$$

We need to find a unique

$$
[\xi]_{h} \in \mathcal{H} \text { top }_{\bullet}\left(\left(X, x_{0}\right) \vee\left(Y, y_{0}\right),\left(Z, z_{0}\right)\right)
$$

such that the following diagrams commute in $\mathcal{H}$ top $\bullet$

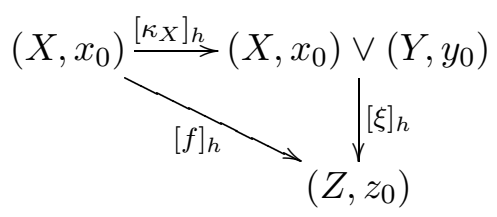

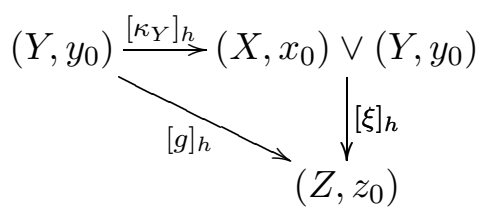

Define $\xi \in \operatorname{top}_{\bullet}\left(\left(X, x_{0}\right) \vee\left(Y, y_{0}\right),\left(Z, z_{0}\right)\right)$ as follows:

$$
\xi((x, y)):= \begin{cases}f(x) & \text { if } y=y_{0}, \\ g(y) & \text { if } x=x_{0}\end{cases}
$$

where $f$ is any element of $[f]_{h}$ and $g$ is any element of $[g]_{h}$.

To prove that (36) commutes it is enough to show that $\xi \circ \kappa_{X}$ and $f$ are in the same base point preserving homotopy class. The required homotopy is defined as

$$
h(x, \cdot):=f(x) \quad \text { for any } x \in X
$$

then

$$
\begin{aligned}
h(x, 0) & =f(x), \\
h(x, 1) & =f(x)=\xi\left(\left(x, y_{0}\right)\right)=\xi \circ \kappa_{X}(x), \\
h\left(x_{0}, t\right) & =f\left(x_{0}\right)=z_{0} \quad \text { for any } t \in I .
\end{aligned}
$$

Similarly one can show that diagram (37) commutes. It is easy to show that such a $\xi$ is unique up to base point preserving homotopy. 
The following is an immediate consequence of Theorems 9 and 8 .

Proposition 10. Let

$$
\left(\left(X, x_{0}\right),\left[a_{X}\right]_{h}\right),\left(\left(Y, y_{0}\right),\left[a_{Y}\right]_{h}\right) \in S z\left(\mathcal{H} t o p_{\bullet}\right),
$$

where $a_{X} \in \operatorname{top}_{\bullet}\left(\left(X, x_{0}\right),\left(X, x_{0}\right)\right)$ and $a_{Y} \in \operatorname{top}_{\bullet}\left(\left(Y, y_{0}\right),\left(Y, y_{0}\right)\right)$. Then the coproduct of the objects $\left(\left(X, x_{0}\right),\left[a_{X}\right]_{h}\right)$ and $\left(\left(Y, y_{0}\right),\left[a_{Y}\right]_{h}\right)$ is the pair

$$
\left(\left(\left(X, x_{0}\right) \vee\left(Y, y_{0}\right),[\alpha]_{h}\right),\left\{\left[\left[\kappa_{X}\right]_{h}, 0\right]_{\equiv},\left[\left[\kappa_{Y}\right]_{h}, 0\right]_{\equiv}\right\}\right),
$$

where $\left(X, x_{0}\right) \vee\left(Y, y_{0}\right)$ is defined by $(33)$, the morphism

$$
[\alpha]_{h}:=\left[\left(\kappa_{X} \circ a_{X}\right) \vee\left(\kappa_{Y} \circ a_{Y}\right)\right]_{h}
$$

is defined as the pointed homotopy class of

$$
\left(\kappa_{X} \circ a_{X}\right) \vee\left(\kappa_{Y} \circ a_{Y}\right)((x, y)):= \begin{cases}\kappa_{X} \circ a_{X}(x) & \text { if } y=y_{0}, \\ \kappa_{Y} \circ a_{Y}(y) & \text { if } x=x_{0}\end{cases}
$$

and the associated inclusions are the Szymczak classes of the pointed homotopy classes of the maps $\kappa_{X}$ and $\kappa_{Y}$ defined respectively by (34) and (35).

5. Additivity of the Szymczak index. Before we proceed to the main theorem of this paper let us prove a lemma.

LEMMA 11. Let $S$ and $\widetilde{S}$ be two isolated invariant sets for $f$ such that

$$
S \cap \widetilde{S}=\emptyset .
$$

Then there exist isolating neighborhoods $M, N$ of $S$ and $\widetilde{S}$ respectively satisfying the conditions

$$
M \cap N=\emptyset, \quad f(M) \cap N=\emptyset, \quad M \cap f(N)=\emptyset .
$$

Proof. The condition $M \cap N=\emptyset$ is easy to satisfy as both $S$ and $\widetilde{S}$ are compact and disjoint.

Consider isolating neighborhoods $M_{m} \subset M$ of $S$ (where $m \in \mathbb{N}$ ) and $N$ of $\widetilde{S}$ such that

$$
M_{m} \cap N=\emptyset \quad \text { and } \quad M_{m+1} \subset M_{m} \quad \text { for } m \in \mathbb{N} .
$$

Moreover assume that

$$
\bigcap_{m \in \mathbb{N}} M_{m}=S
$$

and

$$
f\left(M_{m}\right) \cap N \neq \emptyset \quad \text { for any } m \in \mathbb{N} .
$$

By (46) we can find a sequence $x_{m} \in M_{m}$ such that

$$
f\left(x_{m}\right) \in N \quad \text { for any } m \in \mathbb{N} \text {. }
$$

By (45) we can choose a subsequence of $\left\{x_{m}\right\}$ converging to a point $s \in S$. Because $S$ is invariant, also $f(s) \in S$. From the continuity of $f$, 
compactness of $N$ and (47) we find that $f(s) \in N$ as well and therefore $S \cap N \neq \emptyset$. This contradicts the assumption $M_{m} \cap N=\emptyset$.

Choosing from the isolating neighborhoods that satisfy the first two conditions in (43), we similarly prove that $M$ and $N$ can be chosen such that also the last formula of (43) is satisfied.

Now we are in a position to prove the main result of this paper, namely the additivity of the Szymczak index.

THEOREM 12. Let $S$ and $\widetilde{S}$ be two disjoint isolated invariant sets for $f$. Then the following objects are isomorphic in $S z\left(\mathcal{H}\right.$ top $\left._{\bullet}\right)$ :

$$
C(S, f) \vee C(\widetilde{S}, f) \simeq C(S \cup \widetilde{S}, f) .
$$

Proof. By Lemma 11 there exist isolating neighborhoods $M, N$ of $S$ and $\widetilde{S}$ respectively satisfying

$$
M \cap N=\emptyset
$$

and

$$
f(M) \cap N=\emptyset \text { and } M \cap f(N)=\emptyset .
$$

Notice first that the right hand side of (48) makes sense, because $S \cup \widetilde{S}$ is an isolated invariant set, in an isolating neighborhood $M \cup N$.

Let $P=\left(P_{1}, P_{2}\right)$ and $Q=\left(Q_{1}, Q_{2}\right)$ be index pairs respectively for $S$ and $\widetilde{S}$ such that $P_{1} \subset M$ and $Q_{1} \subset N$. Let us first show that the pair $R:=\left(R_{1}, R_{2}\right)$ defined as

is an index pair for $S \cup \widetilde{S}$.

$$
R_{1}:=P_{1} \cup Q_{1}, \quad R_{2}:=P_{2} \cup Q_{2}
$$

Since $P_{2} \subset P_{1}, Q_{2} \subset Q_{1}$ and also $P_{1} \cap Q_{1}=\emptyset$ by the choice of $P_{1}, Q_{1}$ and (49),

$$
\operatorname{cl}\left(R_{1} \backslash R_{2}\right)=\operatorname{cl}\left(P_{1} \backslash P_{2}\right) \cup \operatorname{cl}\left(Q_{1} \backslash Q_{2}\right) \subset M \cup N
$$

and

$$
\operatorname{Inv}\left(\operatorname{cl}\left(R_{1} \backslash R_{2}\right), f\right) \subset \operatorname{Inv}(M \cup N, f)=S \cup \widetilde{S} .
$$

The inverse inclusion is easy to see.

Obviously

$$
S \cup \widetilde{S} \subset \operatorname{int}\left(P_{1} \backslash P_{2}\right) \cup \operatorname{int}\left(Q_{1} \backslash Q_{2}\right) \subset \operatorname{int}\left(R_{1} \backslash R_{2}\right),
$$

which completes the proof of property (a) from Definition 3 .

To prove (b) we need the finer choice of isolating neighborhoods which is guaranteed by $(50)$.

Consider $x \in P_{2} \cup Q_{2}$. We will prove that if $x \in P_{2}$ then $f(x) \notin R_{1} \backslash R_{2}$. Similarly we argue for $x \in Q_{2}$.

Because $x \in P_{2}$ by property (b) for $P$ we have

$$
f(x) \notin P_{1} \backslash P_{2} .
$$


Since $P_{2} \subset M$ and $Q_{1} \backslash Q_{2} \subset N$, by the first property in (50) we obtain $f\left(P_{2}\right) \cap\left(Q_{1} \backslash Q_{2}\right)=\emptyset$, and so

$$
f(x) \notin Q_{1} \backslash Q_{2} .
$$

From (53) and (54) we infer that $f(x) \notin R_{1} \backslash R_{2}$.

Property (c) for $R$ is a straightforward consequence of property (c) for $P$ and $Q$.

So we have shown that $R$ is an index pair for $S \cup \widetilde{S}$.

To prove (48) it is enough to show that the following objects are isomorphic in $S z\left(\mathcal{H}\right.$ top $\left._{\bullet}\right)$ :

$$
\left(P_{1} / P_{2},\left[f_{P}\right]_{h}\right) \vee\left(Q_{1} / Q_{2},\left[f_{Q}\right]_{h}\right) \simeq\left(R_{1} / R_{2},\left[f_{R}\right]_{h}\right) .
$$

According to Proposition 10 the left hand side of (55) is equal to

$$
\left(P_{1} / P_{2} \vee Q_{1} / Q_{2},\left[\left(\kappa_{P} \circ f_{P}\right) \vee\left(\kappa_{Q} \circ f_{Q}\right)\right]_{h}\right),
$$

where $\kappa_{P}: P_{1} / P_{2} \rightarrow P_{1} / P_{2} \vee Q_{1} / Q_{2}$ and $\kappa_{Q}: Q_{1} / Q_{2} \rightarrow P_{1} / P_{2} \vee Q_{1} / Q_{2}$ are defined as follows:

$$
\begin{array}{ll}
\kappa_{P}([x]):=\left[\left(x, q_{2}\right)\right] & \text { for } x \in P_{1} / P_{2}, \\
\kappa_{Q}([x]):=\left[\left(p_{2}, x\right)\right] & \text { for } x \in Q_{1} / Q_{2} .
\end{array}
$$

By the definition (33) of the coproduct in category $\mathcal{H}$ top • the object $P_{1} / P_{2} \vee Q_{1} / Q_{2}$ is equal to

$$
\left(\left(\left(P_{1} \backslash P_{2}\right) \times\left\{q_{2}\right\}\right) \cup\left(\left\{p_{2}\right\} \times\left(Q_{1} \backslash Q_{2}\right)\right) \cup\left\{\left(p_{2}, q_{2}\right)\right\},\left(p_{2}, q_{2}\right)\right) .
$$

Notice first that

$$
\begin{gathered}
\kappa_{P} \circ f_{P}([x])= \begin{cases}{\left[\left(f(x), q_{2}\right)\right]} & \text { if } x, f(x) \in P_{1} \backslash P_{2}, \\
{\left[\left(p_{2}, q_{2}\right)\right]} & \text { otherwise, }\end{cases} \\
\kappa_{Q} \circ f_{Q}([y])= \begin{cases}{\left[\left(p_{2}, f(y)\right)\right]} & \text { if } y, f(y) \in Q_{1} \backslash Q_{2}, \\
{\left[\left(p_{2}, q_{2}\right)\right]} & \text { otherwise. }\end{cases}
\end{gathered}
$$

To simplify notation put

$$
\xi:=\left(\kappa_{P} \circ f_{P}\right) \vee\left(\kappa_{Q} \circ f_{Q}\right) .
$$

According to formulas (41) and (60), (61) we obtain

$$
(63) \xi([(x, y)])= \begin{cases}{\left[\left(f(x), q_{2}\right)\right]} & \text { if } y=q_{2} \text { and } x, f(x) \in P_{1} \backslash P_{2}, \\ {\left[\left(p_{2}, q_{2}\right)\right]} & \text { if } y=q_{2} \text { and }\left(x \notin P_{1} \backslash P_{2}\right. \\ & \left.\quad \text { or } f(x) \notin P_{1} \backslash P_{2}\right), \\ {\left[\left(p_{2}, f(y)\right)\right]} & \text { if } x=p_{2} \text { and } y, f(y) \in Q_{1} \backslash Q_{2}, \\ {\left[\left(p_{2}, q_{2}\right)\right]} & \text { if } x=p_{2} \text { and }\left(y \notin Q_{1} \backslash Q_{2}\right.\end{cases}
$$

So to prove (55) we should find an isomorphism between the objects $\left(P_{1} / P_{2} \vee Q_{1} / Q_{2},[\xi]_{h}\right)$ and $\left(R_{1} / R_{2},\left[f_{R}\right]_{h}\right)$ in the Szymczak category. 
Define first a map

$$
\chi \in \operatorname{top}_{\bullet}\left(R_{1} / R_{2}, P_{1} / P_{2} \vee Q_{1} / Q_{2}\right)
$$

as follows:

$$
\chi([x]):= \begin{cases}{\left[\left(x, q_{2}\right)\right]} & \text { if } x \in P_{1} \backslash P_{2}, \\ {\left[\left(p_{2}, x\right)\right]} & \text { if } x \in Q_{1} \backslash Q_{2}, \\ {\left[\left(p_{2}, q_{2}\right)\right]} & \text { if } x=r_{2} .\end{cases}
$$

Note that $\chi$ is a well defined continuous base point preserving map.

Moreover it has a continuous inverse

$$
\chi^{-1} \in \operatorname{top}_{\bullet}\left(P_{1} / P_{2} \vee Q_{1} / Q_{2}, R_{1} / R_{2}\right),
$$

defined as follows:

$$
\chi^{-1}([(x, y)])= \begin{cases}{[x]} & \text { if } x \in P_{1} \backslash P_{2} \text { and } y=q_{2} \\ {[y]} & \text { if } x=p_{2} \text { and } y \in Q_{1} \backslash Q_{2} \\ {\left[r_{2}\right]} & \text { if }(x, y)=\left(p_{2}, q_{2}\right)\end{cases}
$$

It is straightforward to verify that $\chi^{-1}$ is in fact inverse to $\chi$ in the category top. We will show that

$$
\left[[\chi]_{h}, 0\right]_{\equiv} \in S z\left(\mathcal{H} \text { top }_{\bullet}\right)\left(\left(R_{1} / R_{2},\left[f_{R}\right]_{h}\right),\left(P_{1} / P_{2} \vee Q_{1} / Q_{2},[\xi]_{h}\right)\right)
$$

is the isomorphism required by $(55)$.

First we need to prove that $[\chi]_{h}$ is an appropriate morphism in Endo $\left(\mathcal{H} t_{o p} \bullet\right)$. To do this we have to prove that the diagram (67) below commutes up to base point preserving homotopy; in fact we will prove that it actually commutes in top.

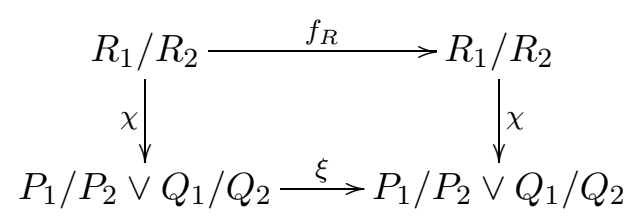

We have

$$
\begin{aligned}
\chi \circ f_{R}([x]) & = \begin{cases}\chi([f(x)]) & \text { if } x, f(x) \in R_{1} \backslash R_{2}, \\
\chi\left(\left[r_{2}\right]\right) & \text { otherwise, }\end{cases} \\
& = \begin{cases}{\left[\left(f(x), q_{2}\right)\right]} & \text { if } x \in R_{1} \backslash R_{2} \text { and } f(x) \in P_{1} \backslash P_{2}, \\
{\left[\left(p_{2}, f(x)\right)\right]} & \text { if } x \in R_{1} \backslash R_{2} \text { and } f(x) \in Q_{1} \backslash Q_{2}, \\
{\left[\left(p_{2}, q_{2}\right)\right]} & \text { otherwise, }\end{cases} \\
& = \begin{cases}{\left[\left(f(x), q_{2}\right)\right]} & \text { if } x, f(x) \in P_{1} \backslash P_{2}, \\
{\left[\left(p_{2}, f(x)\right)\right]} & \text { if } x, f(x) \in Q_{1} \backslash Q_{2}, \\
{\left[\left(p_{2}, q_{2}\right)\right]} & \text { otherwise. }\end{cases}
\end{aligned}
$$

To justify the last equality note that neither of the conditions $x \in P_{1} \backslash P_{2}$ and $f(x) \in Q_{1} \backslash Q_{2}, \quad x \in Q_{1} \backslash Q_{2}$ and $f(x) \in P_{1} \backslash P_{2}$ can be satisfied by any $x$. 
Now write

$$
\begin{aligned}
& \xi \circ \chi([x])= \begin{cases}\xi\left(\left[\left(x, q_{2}\right)\right]\right) & \text { if } x \in P_{1} \backslash P_{2}, \\
\xi\left(\left[\left(p_{2}, x\right)\right]\right) & \text { if } x \in Q_{1} \backslash Q_{2}, \\
\xi\left(\left[\left(p_{2}, q_{2}\right)\right]\right) & \text { if } x=r_{2},\end{cases} \\
&= \begin{cases}{\left[\left(f(x), q_{2}\right)\right]} & \text { if } x, f(x) \in P_{1} \backslash P_{2}, \\
{\left[\left(p_{2}, q_{2}\right)\right]} & \text { if } x \in P_{1} \backslash P_{2} \text { and } f(x) \notin P_{1} \backslash P_{2}, \\
{\left[\left(p_{2}, f(x)\right)\right]} & \text { if } x, f(x) \in Q_{1} \backslash Q_{2}, \\
{\left[\left(p_{2}, q_{2}\right)\right]} & \text { if } x \in Q_{1} \backslash Q_{2} \text { and } f(x) \notin Q_{1} \backslash Q_{2}, \\
{\left[\left(p_{2}, q_{2}\right)\right]} & \text { if } x \in P_{2} \cup Q_{2} \text { or } \notin P_{1} \cup Q_{1},\end{cases} \\
&= \begin{cases}{\left[\left(f(x), q_{2}\right)\right]} & \text { if } x, f(x) \in P_{1} \backslash P_{2}, \\
{\left[\left(p_{2}, f(x)\right)\right]} & \text { if } x, f(x) \in Q_{1} \backslash Q_{2}, \\
{\left[\left(p_{2}, q_{2}\right)\right]} & \text { otherwise. }\end{cases}
\end{aligned}
$$

To justify the last equality notice that the condition under which $\xi \circ \chi([x])=$ $\left[\left(p_{2}, q_{2}\right)\right]$ excludes all the other cases.

Comparing the formulas for $\chi \circ f_{R}$ and $\xi \circ \chi$ proves that diagram (67) commutes.

It can be easily noticed that by reversing the $\chi$ arrows in (67), we prove that $\left[\left[\chi^{-1}\right]_{h}, 0\right]_{\equiv}$ is also a morphism in $S z\left(\mathcal{H}\right.$ top $\left._{\bullet}\right)$.

Finally,

$$
\begin{aligned}
{\left[[\chi]_{h}, 0\right]_{\equiv \star}\left[\left[\chi^{-1}\right]_{h}, 0\right]_{\equiv} } & =\left[[\chi]_{h} \bullet\left[\chi^{-1}\right]_{h}, 0\right]_{\equiv}=\left[\left[\chi \circ \chi^{-1}\right]_{h}, 0\right]_{\equiv} \\
& =\left[\left[\operatorname{id}_{P_{1} / P_{2} \vee Q_{1} / Q_{2}}\right]_{h}, 0\right]_{\equiv},
\end{aligned}
$$

and similarly

$$
\left[\left[\chi^{-1}\right]_{h}, 0\right]_{\equiv \star}\left[[\chi]_{h}, 0\right]_{\equiv}=\left[\left[\operatorname{id}_{R_{1} / R_{2}}\right]_{h}, 0\right]_{\equiv},
$$

which completes the proof of (55).

\section{References}

[1] C. Conley, Isolated Invariant Sets and the Morse Index, CBMS Reg. Conf. Ser. Math. 38, Amer. Math. Soc. Providence, RI, 1978.

[2] S. MacLane, Categories for the Working Mathematician, Grad. Texts in Math. 5, Springer, 1971.

[3] M. Mrozek, Normal functors and retractors in categories of endomorphisms, Univ. Iagell. Acta Math. 29 (1992), 181-198.

[4] - Shape index and other indices of Conley type for locally compact Hausdorff spaces, Fund. Math. 145 (1994), 15-37.

[5] B. Pareigis, Categories and Functors, Pure and Appl. Math. 39, Academic Press, New York, 1970.

[6] K. Stolot, Homotopy Conley index for discrete multivalued dynamical systems, $\mathrm{PhD}$ dissertation, Jagiellonian Univ., Kraków, 2003 (in Polish).

[7] J. Szybowski, Conley index over the base space for discrete semidynamical systems, PhD dissertation, Jagiellonian Univ., Kraków, 2002 (in Polish). 
[8] A. Szymczak, The Conley index for decompositions of isolated invariant sets, Fund. Math. 148 (1995), 71-90.

[9] - , The Conley index for discrete semidynamical systems, Topology Appl. 66 (1995), 215-240.

Faculty of Applied Mathematics

AGH University of Science and Technology

Al. Mickiewicza 30

30-059 Kraków, Poland

E-mail: stolot@uci.agh.edu.pl

Reçu par la Rédaction le 26.10.2004

Révisé le 4.5.2005 